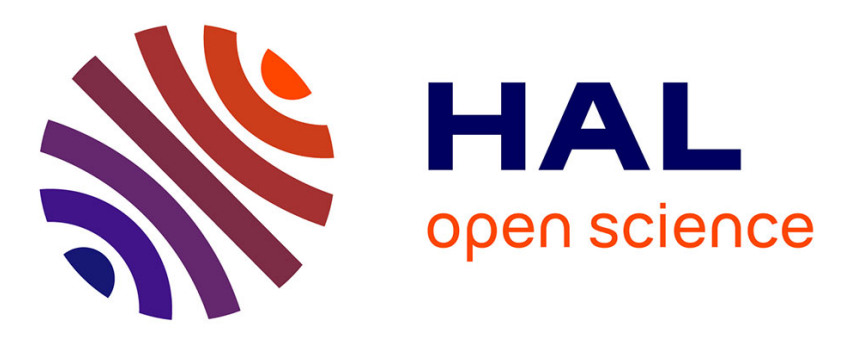

\title{
Energy-Efficient Cooperative Routing in BER Constrained Multihop Networks
}

Behrouz Maham, Merouane Debbah, Are Hjorungnes

\section{To cite this version:}

Behrouz Maham, Merouane Debbah, Are Hjorungnes. Energy-Efficient Cooperative Routing in BER Constrained Multihop Networks. Frontiers of Computer Science in China, 2009, 3 (2), pp. 263-271. hal-00446954

\section{HAL Id: hal-00446954 \\ https://hal-centralesupelec.archives-ouvertes.fr/hal-00446954}

Submitted on 13 Jan 2010

HAL is a multi-disciplinary open access archive for the deposit and dissemination of scientific research documents, whether they are published or not. The documents may come from teaching and research institutions in France or abroad, or from public or private research centers.
L'archive ouverte pluridisciplinaire HAL, est destinée au dépôt et à la diffusion de documents scientifiques de niveau recherche, publiés ou non, émanant des établissements d'enseignement et de recherche français ou étrangers, des laboratoires publics ou privés. 


\title{
Energy-Efficient Cooperative Routing in BER Constrained Multihop Networks
}

\author{
Behrouz Maham ${ }^{1,2}$, Mérouane Debbah ${ }^{3}$, and Are Hjørungnes ${ }^{1}$ \\ ${ }^{1}$ UNIK - University Graduate Center, University of Oslo, Norway \\ ${ }^{2}$ Department of Electrical Engineering, Stanford University, Stanford, USA \\ ${ }^{3}$ Alcatel-Lucent Chair on Flexible Radio, SUPÉLEC, Gif-sur-Yvette, France \\ Email: behrouz@unik.no, merouane.debbah@supelec.fr, arehj@unik.no
}

\begin{abstract}
Due to the limited energy supplies of nodes, in many applications like wireless sensor networks energy-efficiency is crucial for extending the lifetime of these networks. We study the routing problem for multihop wireless ad hoc networks based on cooperative transmission. The source node wants to transmit messages to a single destination. Other nodes in the network may operate as relay nodes. In this paper, we propose a cooperative multihop routing for the purpose of power savings, constrained on a required bit error rate (BER) at the destination. We derive analytical results for line and grid network topologies. It is shown that energy savings of $100 \%$ are achievable in line and grid networks with a large number of nodes for BER $=10^{-4}$ constraint at the destination.
\end{abstract}

\section{INTRODUCTION}

Energy saving is one of the main objectives of routing algorithms for different wireless networks such as mobile ad hoc networks [1] and sensor networks [2]. In [3], it was shown that in some wireless networks such as ad hoc networks, nodes spend most of their power in communication, either sending their own data or relaying other nodes' data. In addition to saving more energy, selected routes may guarantee certain Quality of Service (QoS). QoS routing is of great importance to some wireless applications (e.g. multimedia applications) [4]. Recently, there have been much interest in studying the interaction between the various network layers, which is known in the literature as cross-layer design [5]. In particular, the physical information about the wireless medium can be provided to the upper layers in order to provide efficient scheduling, routing, resource allocation, and flow control algorithms.

Space-time codes provide diversity and coding gains in multiple antenna systems over fading channels. In ad-hoc or distributed large scale wireless networks, nodes are often constrained in hardware complexity and size, which makes multiple antenna systems impractical for certain applications. Cooperative diversity schemes [6], [7] have been introduced in an effort to overcome this limitation. Cooperative techniques allow a collection of radios to relay signals amongst each other, effectively creating a virtual antenna array, which combat multipath fading in wireless channels. This makes cooperative techniques attractive for deployment in cellular

This work was supported by the Research Council of Norway through the project 176773/S10 entitled "Optimized Heterogeneous Multiuser MIMO Networks - OptiMO" and the AURORA project entitled "Communications under uncertain topologies". mobile devices as well as in ad-hoc mobile networks. Several cooperation strategies with different relaying techniques, including amplify-and-forward (AF), decode-and-forward (DF), and selective relaying (SR), have been studied in Laneman et al.'s seminal paper [8].

Energy consumption in multihop wireless networks is a crucial issue that needs to be addressed at all the layers of communication system, from the hardware up to the application. In this paper, we focus on energy savings in routing problem in which messages may be transmitted via multiple radio hops. After substantial research efforts in the last several years, routing for multihop wireless networks becomes a well-understood and broadly investigated problem [9], [10]. Nevertheless, with the emergence of new multiple antennas technology, existing routing solutions in the traditional radio transmission model are not efficient anymore. For instance, it is feasible to coordinate the multiple transmissions from multiple transmitters to one receiver simultaneously. As a result, transmitting signals with the same channel from several different nodes to the same receiver simultaneously are not considered collision but instead could be combined at the receiver to obtain stronger signal strength. In [11], the concept of multihop diversity is introduced where the benefits of spatial diversity are achieved from the concurrent reception of signals that have been transmitted by multiple previous terminals along the single primary route. This scheme exploits the broadcast nature of wireless networks where the communications channel is shared among multiple terminals. On the other hand, the routing problem in the cooperative radio transmission model is studied in [12], where it is allowed that multiple nodes along a path coordinate together to transmit a message to the next hop as long as the combined signal at the receiver satisfies a given SNR threshold value. Also in [13], some cooperation-based routing algorithms are proposed, which makes full use of the cooperative communications while constructing the minimum-power route.

In this paper, a cooperative multihop routing is proposed for Rayleigh fading channels. The investigated system can achieve considerable power savings compared to non-cooperative multihop transmission, when there is a bit error rate (BER) QoS requirement at the destination node. We derive a simple closedform solution for power allocation among the transmitting nodes at each phase. Simulation results show that, using the proposed power allocation strategies, considerable gains are 


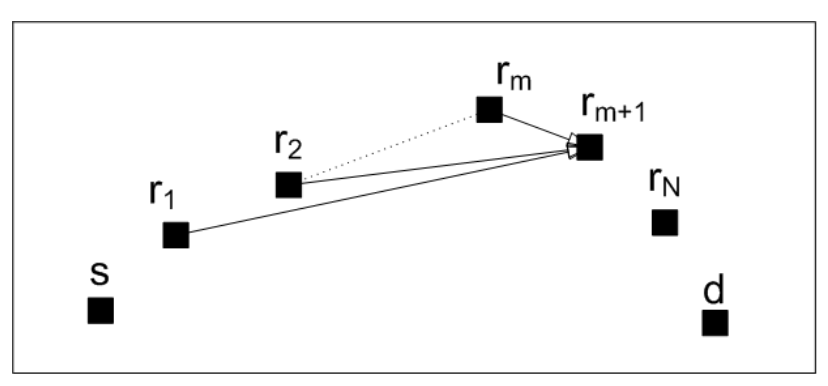

Fig. 1. Wireless multihop network under $m$-cooperation.

obtained comparing to the non-cooperative multihop transmission.

This paper is organized as follows. In Section II, the system model is given. The formulation of link costs based on BER constraint at the receiving node over Rayleigh fading environment is presented in Section III. In Section IV, the proposed link cost formulation is employed for the purpose of energy savings in cooperative routing. In Section V, the overall performance of the system are presented for classical line and grid networks. Finally, conclusion is presented in Section VI.

\section{System Model And Protocol Description}

We consider an arbitrary $N$-relay wireless network, where information is to be transmitted from a source to a destination. Due to the broadcast nature of the wireless channel, some relays can overhear the transmitted information, and thus, can cooperate with the source to send its data. The wireless link between any two nodes in the network is modeled as a Rayleigh fading narrowband channel. The channel fades for different links are assumed to be statistically independent. The additive noise at all receiving terminals is modeled as zero-mean complex Gaussian random variables with variance $\mathcal{N}_{0}$. For medium access, the relays are assumed to transmit over orthogonal channels, thus no interrelay interference is considered in the signal model.

Following [12], we also assume that each transmission is either a broadcast transmission where a single node is transmitting the information, and the information is received by multiple nodes, or a cooperative transmission where multiple nodes simultaneously send the information to a single receiver. Various scenarios for the cooperation among the relays can be implemented. A general cooperation scenario, $m$-cooperation, $(1 \leq m \leq N)$, can be implemented in which each relay combines the signals received from the previous relays and along with that received from the source.

For a general scheme $m$-cooperation, $(1 \leq m \leq N)$, each receiving node decodes the information after combining the signals received from the previous $m$ transmitting nodes. Fig. 1 shows a wireless multihop network consisting of a source node $s, N$ relays, and a destination node $d$, which is operating under $m$-cooperation scenario. The cooperation protocol has $N+1$ phases. In Phase 1, the source transmits the information, and the received signal at the destination and the $i$ th relay can be modeled, respectively, as

$$
\begin{aligned}
y_{0} & =\sqrt{P_{0}} f_{0} s+w_{0}, \\
y_{0, i} & =\sqrt{P_{0}} f_{0, i} s+v_{i},
\end{aligned}
$$

where $P_{0}$ is the average total transmitted symbol energy of the source, since we assume the information bearing symbols $s$ 's have zero-mean and unit variance, $w_{0}$ and $v_{i}$ are complex zero-mean white Gaussian noise, and $f_{i, j}, i=0,1, \ldots, N$, $j=1,2 \ldots, N+1$, are complex Gaussian random variables with zero-mean and variances $\sigma_{i, j}^{2}$, respectively. In Phase 2, relay nodes are sorted based on their received received SNR, such that relay 1 has the highest received SNR. Generally, in Phase $n, 2 \leq n \leq N+1$, the previous $\min \{m, n\}$ nodes are transmitting their signal toward the next node. Similar to [12], we assume that transmitters are able to adjust their phases in such a way that the received signal at the $n$th receiving node in Phase $n$ is

$y_{n}=\sqrt{P_{0}}\left|f_{0, n}\right| u(m-n) s+\sum_{i=\max (1, n-m)}^{n-1} \sqrt{P_{i}}\left|f_{i, n}\right| \hat{s}_{i}+v_{n}$,

where the function $u(x)=1$, when $x \geq 0$, and otherwise is zero and the symbol $\hat{s}_{i}$ is re-encoded symbol at the $i$ th relay.

\section{BER-BASED LINK COST FORMULATION}

In this section, our objective is to find the optimal power allocation required for successful transmission from a set of transmitting nodes to a set of receivers. In order to derive explicit expressions for the link costs, we consider three distinct cases described as follows.

\section{A. Point-to-Point Link Cost}

The simplest case is the case where only one node is transmitting within a time slot to a single target node. For decoding the message reliably, the BER must be less than the threshold value $\mathrm{BER}_{\max }$.

Assuming a Rayleigh fading link with variance of $\sigma_{0}^{2}$ in the network, $M$-PSK or $M$-QAM modulations, and coherent detection, the average probability of error can be obtain as [14, Eq. (6)],

$$
P_{e}^{b}=\frac{c}{\pi}\left(1-\sqrt{\frac{g P_{0} \sigma_{0}^{2}}{2 \mathcal{N}_{0}+g P_{0} \sigma_{0}^{2}}}\right),
$$

where the parameters $c$ and $g$ are dependent on the modulation type. Using (4), the minimum required power, and hence, the point-to-point link cost is given by

$$
\mathcal{C}\left(\operatorname{tx}_{1}, \mathrm{rx}_{1}\right)=P_{0}=\frac{2 \mathcal{N}_{0}}{g \sigma_{0}^{2}} \frac{1}{\left(1-\frac{2 \mathrm{BER}_{\max }}{c}\right)^{2}}-1
$$

where $\mathrm{tx}_{1}$ and $\mathrm{rx}_{1}$ denote the transmitter and receiver nodes, respectively. Since $\mathrm{BER}_{\max } \ll 1$, the link cost in (5) can be approximated as

$$
\mathcal{C}\left(\mathrm{tx}_{1}, \mathrm{rx}_{1}\right) \approx \frac{\mathcal{N}_{0}}{g \sigma_{0}^{2}} \frac{c}{2 \mathrm{BER}_{\max }}
$$




\section{B. Point-to-Multipoint Link Cost}

In this case, we assume a transmitter node $\mathrm{tx}_{1}$ broadcast its information toward a set of receiving nodes $\mathrm{Rx}=$ $\left\{\mathrm{rx}_{1}, \mathrm{rx}_{2}, \ldots, \mathrm{rx}_{m}\right\}$. Assuming that omnidirectional antennas are used, the signal transmitted by the node $\mathrm{tx}_{1}$ is received by all nodes within a transmission radius proportional to the transmission power. Hence, a broadcast link can be treated as a set of point-to-point links, and the cost of reaching a set of nodes is the maximum of the costs for reaching each of the nodes in the target set. Thus, the minimum power required for the broadcast transmission, denoted by $\mathcal{C}\left(\mathrm{tx}_{1}, \mathrm{Rx}\right)$, is given by

$$
\mathcal{C}\left(\mathrm{tx}_{1}, \mathrm{Rx}\right)=\max \left\{\mathcal{C}\left(\mathrm{tx}_{1}, \mathrm{rx}_{1}\right), \mathcal{C}\left(\mathrm{tx}_{1}, \mathrm{rx}_{2}\right), \ldots, \mathcal{C}\left(\mathrm{tx}_{1}, \mathrm{rx}_{m}\right)\right\}
$$

where $\mathcal{C}\left(\mathrm{tx}_{1}, \mathrm{rx}_{i}\right)$ is found from (5).

\section{Multipoint-to-Point Cooperative Link Cost}

In this case, a set of multiple nodes $\mathrm{Tx}=$ $\left\{\mathrm{tx}_{1}, \mathrm{tx}_{2}, \ldots, \mathrm{tx}_{m}\right\}$ cooperate to transmit the same information to a single receiver node $\mathrm{rx}_{1}$. Assuming coherent detection at the receiving node, the signals simply add up at the receiver, and acceptable decoding is possible as long as the received BER becomes less than $\mathrm{BER}_{\text {max }}$.

Now, we are going to derive a tractable BER formula at the receiving node $\mathrm{rx}_{1}$, which leads to a closed-form power allocation strategy among the cooperative nodes. Therefore, we use the approach proposed in [15] to derive the BER expressions for the high SNR regime. That is [15, Eq. (10)]

$$
P_{e}^{b} \simeq \frac{c \prod_{i=1}^{t+1}(2 i-1)}{2(t+1) g^{t+1} t !} \frac{\partial^{t} p_{\gamma}(0)}{\partial \gamma^{t}}
$$

where $\frac{\partial^{t} p_{\gamma}(0)}{\partial \gamma^{t}}$ is the $t$ th order derivative of the pdf of the equivalent channel, and the derivatives of $p_{\gamma}(\gamma)$ up to order $(t-1)$ are supposed to be zero. Using (3), the received SNR at the receiving node can be written as $\gamma=\sum_{i=1}^{m} \gamma_{i}$, where $\gamma_{i}=$ $\frac{P_{i}\left|f_{i}\right|^{2}}{\mathcal{N}_{0}}$ with $f_{i}$ denotes the channel between the $i$ th transmitter and the receiving node.

In [15], the following proposition is proposed, which can be used to calculate the BER expression in (8).

Proposition 1: Consider a finite set of nonnegative random variables $\left\{\gamma_{1}, \gamma_{2}, \ldots, \gamma_{m}\right\}$ whose pdfs $p_{1}, p_{2}, \ldots, p_{m}$ have nonzero values at zero, and denote these values as $p_{1}(0), p_{2}(0), \ldots, p_{m}(0)$. If $\gamma=\sum_{i=1}^{m} \gamma_{i}$, then all the derivatives of $p_{\gamma}(\gamma)$ evaluated at zero up to order $m-2$ are zero, while the $(m-1)$ th order derivative is given by

$$
\frac{\partial^{m-1} p_{\gamma}(0)}{\partial \gamma^{m-1}}=\prod_{i=1}^{m} p_{i}(0)
$$

Using Proposition 1 and (8), we get

$$
P_{e}^{b} \approx \frac{c \prod_{i=1}^{m}(2 i-1)}{2 g^{m} m !} \prod_{i=1}^{m} p_{i}(0) .
$$

Hence, using (10) and the fact that the value of an exponential distribution with mean $\frac{P_{i} \sigma_{i}^{2}}{\mathcal{N}_{0}}$ at zero is $\frac{\mathcal{N}_{0}}{P_{i} \sigma_{i}^{2}}$, the average BER expression can be approximated as

$$
P_{e}^{b} \approx \frac{c \prod_{i=1}^{m}(2 i-1)}{2 g^{m} m !} \prod_{i=1}^{m} \frac{\mathcal{N}_{0}}{P_{i} \sigma_{i}^{2}} .
$$

The total transmitted power for the multipoint-to-point case is $\sum_{i=1}^{m} P_{i}$. Therefore, the power allocation problem, which has a required BER constraint on the receiving node, can be formulated as

$$
\begin{array}{ll}
\min & \sum_{i=1}^{m} P_{i} \\
\text { s.t. } & \frac{c}{2 g^{m} m !} \prod_{i=1}^{m} \frac{(2 i-1) \mathcal{N}_{0}}{P_{i} \sigma_{i}^{2}} \leq \mathrm{BER}_{\max }, \\
& P_{i} \geq 0, \text { for } i=1, \ldots, m
\end{array}
$$

Before deriving the optimal solution for the problem given in (12), the following theorem is needed.

Theorem 1: The optimum power allocation $P_{1}^{*}, \ldots, P_{m}^{*}$ in the optimization problem stated in (12) is unique.

Proof: The objective function in (12) is a linear function of the power allocation parameters, and thus, it is a convex function. Hence, it is enough to prove that the first constraint in (12), i.e.,

$$
f\left(P_{1}, \ldots, P_{m}\right)=\frac{c}{2 g^{m} m !} \prod_{i=1}^{m} \frac{(2 i-1) \mathcal{N}_{0}}{P_{i} \sigma_{i}^{2}}-\mathrm{BER}_{\max }
$$

with $D_{f}=\left\{P_{i} \in(0, \infty), i \in\{1, \ldots, m\} \mid f\left(P_{1}, \ldots, P_{m}\right) \leq 0\right\}$, $f: D_{f} \longrightarrow \mathbb{R}$, is a convex function. From [16], it can be verified that $f\left(P_{1}, \ldots, P_{m}\right)$ is a posynomial function, which is a convex function.

The optimal power allocation strategy for high SNRs is found in the following. However, since the approximate BER expression derived in (11) is an upper-bound on BER, this result can be used reliably.

Proposition 2: For the set of $m$ transmitters, which send a common signal toward the destination, the optimum transmit power coefficients in (12) satisfy the following equations

$$
P_{i}=\frac{\Psi(m)}{\mathrm{BER}_{\max }} \frac{\mathcal{N}_{0}}{\sigma_{i}^{2}} \prod_{\substack{k=1 \\ k \neq i}}^{m} \frac{\mathcal{N}_{0}}{P_{k} \sigma_{k}^{2}}, \quad i=1, \ldots, m,
$$

where

$$
\Psi(m)=\frac{c \prod_{i=1}^{m}(2 i-1)}{2 g^{m} m !},
$$

Proof: The Lagrangian of the problem stated in (12) is

$$
L\left(P_{1}, \ldots, P_{m}\right)=\sum_{i=1}^{m} P_{i}+\lambda f\left(P_{1}, \ldots, P_{m}\right) .
$$

For nodes $i=1, \ldots, m$ with nonzero transmitter powers, the Kuhn-Tucker conditions are

$$
\frac{\partial}{\partial P_{i}} L\left(P_{1}, \ldots, P_{m}\right)=1+\lambda \frac{\partial}{\partial P_{i}} f\left(P_{1}, \ldots, P_{m}\right)=0,
$$

where

$$
\frac{\partial}{\partial P_{i}} f\left(P_{1}, \ldots, P_{m}\right)=-\Psi(m) \frac{\mathcal{N}_{0}}{P_{i}^{2} \sigma_{i}^{2}} \prod_{\substack{k=1 \\ k \neq i}}^{m} \frac{\mathcal{N}_{0}}{P_{k} \sigma_{k}^{2}} .
$$


Using (17) and (18), we have

$$
P_{i}^{2}=\lambda \Psi(m) \frac{\mathcal{N}_{0}}{P_{i}^{2} \sigma_{i}^{2}} \prod_{\substack{k=1 \\ k \neq i}}^{m} \frac{\mathcal{N}_{0}}{P_{k} \sigma_{k}^{2}}
$$

for $i=1, \ldots, m$. Since the strong duality condition [16, Eq. (5.48)] holds for convex optimization problems, we have $\lambda f\left(P_{1}, \ldots, P_{m}\right)=0$ for the optimum point. If we assume Lagrange multiplier has a positive value, we have $f\left(P_{1}, \ldots, P_{m}\right)=0$, which is equivalent to

$$
\mathrm{BER}_{\max }=\Psi(m) \prod_{k=1}^{m} \frac{\mathcal{N}_{0}}{P_{k} \sigma_{k}^{2}} .
$$

Dividing both sides of equalities (19) and (20), we can find the Lagrange multiplier as

$$
\lambda=\frac{P_{i}}{\mathrm{BER}_{\max }} .
$$

Substituting $\lambda$ from (21) into (19) we get (14). Moreover, since $P_{i}$ in (14) are positive, the second set of constraints in (12) are satisfied.

Theorem 2: The optimum power allocation $P_{1}^{*}, \ldots, P_{m}^{*}$ in the optimization problem stated in (12) are equal and is expressed as

$$
P_{i}^{*}=\left(\frac{\Psi(m)}{\mathrm{BER}_{\max }} \prod_{k=1}^{m} \frac{\mathcal{N}_{0}}{\sigma_{k}^{2}}\right)^{\frac{1}{m}} .
$$

Proof: In Theorem 1, we have shown, this problem has a unique solution. Now, using Proposition 2, by the fact that the problem in (12) should have a unique solution, we put initial values $P_{1}^{*}, \ldots, P_{m}^{*}$ in (14), and we observe that the closedform solution as (22) is achieved, which satisfies the set of equations in (14).

An interesting property of $P_{i}^{*}$ derived in (22) is that it is just dependent on the product of all path-loss coefficients of links. Therefore, $P_{i}^{*}$ s can be calculated in a decentralized manner by broadcasting the product term from the receiving node toward the transmitting nodes. Using Theorem 2, the resulting cooperative link cost $\mathcal{C}\left(\mathrm{Tx}, \mathrm{rx}_{1}\right)$, defined as the optimal total power, is given by

$$
\mathcal{C}\left(\mathrm{Tx}, \mathrm{rx}_{1}\right)=\sum_{i=1}^{m} P_{i}^{*}=m\left(\frac{\Psi(m)}{\mathrm{BER}_{\max }} \prod_{k=1}^{m} \frac{\mathcal{N}_{0}}{\sigma_{k}^{2}}\right)^{\frac{1}{m}} .
$$

\section{ENERGy SAVINGS Via COOPERATIVE Routing}

The problem of finding the optimal cooperative route from the source node to the destination node can be mapped to a Dynamic Programming (DP) problem [12]. As the network nodes are allowed only to either fully cooperate or broadcast, finding the best cooperative path from the source node to the destination has a special layered structure. In [12], it is shown that in a network with $N+1$ nodes, which has $2^{N}$ nodes in the cooperation graph, standard shortest path algorithms have a complexity of $O\left(2^{N}\right)$. Hence, finding the optimal cooperative route in an arbitrary network becomes computationally intractable for larger networks. For this reason, we restrict the cooperation to nodes along the optimal noncooperative route. That is, at each transmission slot, all nodes that have received the information cooperate to send the information to the next node along the minimum energy noncooperative route [12]. Therefore, with the help of the link cost expressed in Subsections III-A and III-B, the minimum-energy non-cooperative route is first selected, which has $N$ intermediate relays. Then, nodes along the optimal non-cooperative route cooperate to transmit the source information toward the destination. That is, at each transmission slot, all nodes that have received the information cooperate to send the information to the next node along the minimum energy non-cooperative route. In the $n$th transmission slot, the reliable set is $\mathrm{Tx}_{n}=\left\{s, r_{1}, \ldots, r_{n-1}\right\}$, which is including the source node and the previous relays $r_{i}$, $i=1, \ldots, n-1$. The link cost associated with the nodes in $\mathrm{Tx}_{n}$, which cooperate to send the information to the next node $n$, follows from (23), and is given by

$$
\mathcal{C}\left(\operatorname{Tx}_{n}, n\right)=n\left(\frac{\Psi(n)}{\mathrm{BER}_{\max }} \prod_{k=0}^{n-1} \frac{\mathcal{N}_{0}}{\sigma_{k, n}^{2}}\right)^{\frac{1}{n}} .
$$

Note that the $n$th node denotes the $n$th relay when $n \leq N$, and the destination node when $n=N+1$. Therefore, the total transmission power for the cooperative multihop system is

$$
P_{T}(\mathrm{coop})=\sum_{n=1}^{N+1} \mathcal{C}\left(\mathrm{Tx}_{n}, n\right)=\sum_{n=1}^{N+1} n\left(\frac{\Psi(n)}{\mathrm{BER}_{\max }} \prod_{k=0}^{n-1} \frac{\mathcal{N}_{0}}{\sigma_{k, n}^{2}}\right)^{\frac{1}{n}} \text {. }
$$

For the case of $m$-cooperation scheme, in which just previous closest nodes cooperate to transmit along the noncooperative route, $P_{T}$ (cooperative) in (25) can be modified to

$$
\begin{aligned}
P_{T}(m-\text { coop }) & =\sum_{n=1}^{N+1} \mathcal{C}_{m}\left(\operatorname{Tx}_{n}, n\right) \\
& =\sum_{n=1}^{m} n\left(\frac{\Psi(n)}{\mathrm{BER}_{\max }} \prod_{k=0}^{n-1} \frac{\mathcal{N}_{0}}{\sigma_{k, n}^{2}}\right)^{\frac{1}{n}} \\
& +\sum_{n=m+1}^{N+1} m\left(\frac{\Psi(m)}{\mathrm{BER}_{\max }} \prod_{k=n-m}^{n-1} \frac{\mathcal{N}_{0}}{\sigma_{k, n}^{2}}\right)^{\frac{1}{m}} .
\end{aligned}
$$

The energy savings for a cooperative routing strategy relative to the optimal noncooperative strategy is defined as

$$
\text { Energy Savings }=\frac{P_{T}(\text { noncoop })-P_{T}(\text { coop })}{P_{T}(\text { noncoop })},
$$

where $P_{T}$ (coop) is computed in (25) and (26) for the case of full-cooperation and $m$-cooperation routings, respectively. $P_{T}$ (noncoop) denotes the total transmission power for the noncooperative multihop strategy. Using (6), $P_{T}$ (noncoop) can be calculated as

$$
P_{T}(\text { noncoop })=\frac{c}{2 \mathrm{BER}_{\max }} \sum_{n=0}^{N} \frac{\mathcal{N}_{0}}{g \sigma_{n, n+1}^{2}} .
$$

For each of these topologies, we derive the optimal noncooperative route and obtain a lower bound on the optimal energy savings achievable by cooperative routing. The bound 


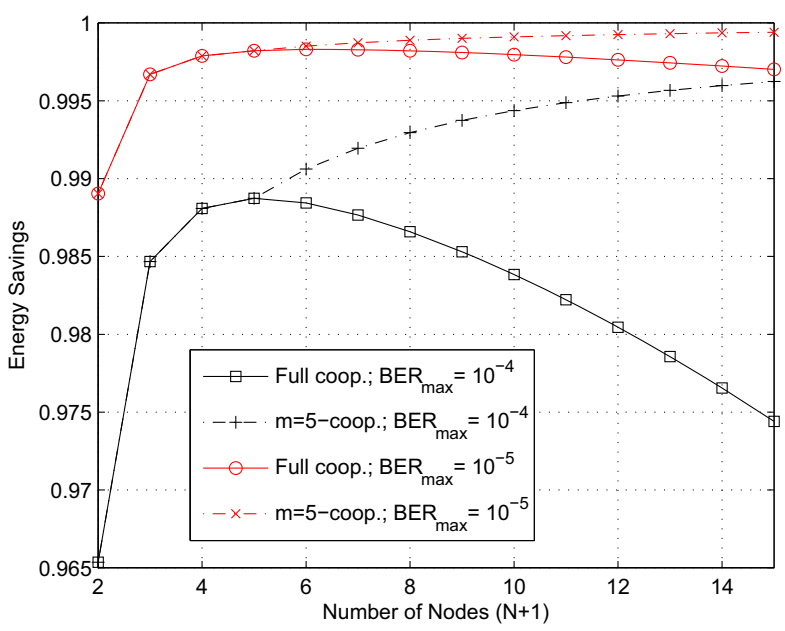

Fig. 2. The average energy savings curves versus the number of transmitting nodes, $(N+1)$, employing full-cooperation and $m$-cooperation with two different $\mathrm{BER}_{\max }$ constraints.

is obtained by deriving analytical expressions for energy savings for a sub-optimal cooperative route, where cooperation is restricted to nodes along the optimal non-cooperative route. That is, at each transmission slot, all nodes that have received the information cooperate to send the information to the next node along the minimum energy non-cooperative route.

\section{A. BER Upper-Bounds at the Destination Node}

In this subsection, we will view the system from our end-toend equivalent BER perspective. That is, we represent $\mathrm{BER}_{\max }$ in each step in terms of the required BER at the destination.

In the case of non-cooperative multihop system in which $N$ relays are in cascade, when BPSK is used, the BER $P_{n}^{b}$ at $n$th node is affected by all previous $n-1$ hops and can be iteratively calculated according to the recursion [17]

$$
P_{n}^{b}=\left(1-P_{n-1}^{b}\right) P_{n-1, n}^{b}+P_{n-1}^{b}\left(1-P_{n-1, n}^{b}\right),
$$

with $P_{0}^{b}=0$, where $P_{n-1, n}^{b}$ is the BER from the $(n-1)$ th node to the $n$th node. The end-to-end BER at the destination is given by using $n=N+1$ in (29). Since the BER at the destination should be less than the required BER QoS, it is enough to consider the upper-bound for the BER. Thus, for any general constellation, the BER can be bounded as $P_{n}^{b} \leq(1-$ $\left.P_{n-1}^{b}\right) P_{n-1, n}^{b}+P_{n-1}^{b}$. Assuming the power allocation strategies derive in Section III, $P_{n}^{b}$ bound can be written as $P_{n}^{b} \leq 1-$ $\left(1-\mathrm{BER}_{\max }\right)^{n}$.

For the case of cooperative routing, the following upperbound can be obtained in the $n$th node

$$
P_{n}^{b} \leq 1-\left[\left(1-P_{n-1, n}^{b}\right) \prod_{i=\max \{1, n-m\}}^{n-1}\left(1-P_{i}^{b}\right)\right]
$$

If the power allocation strategy derived in (22) is used, (30)

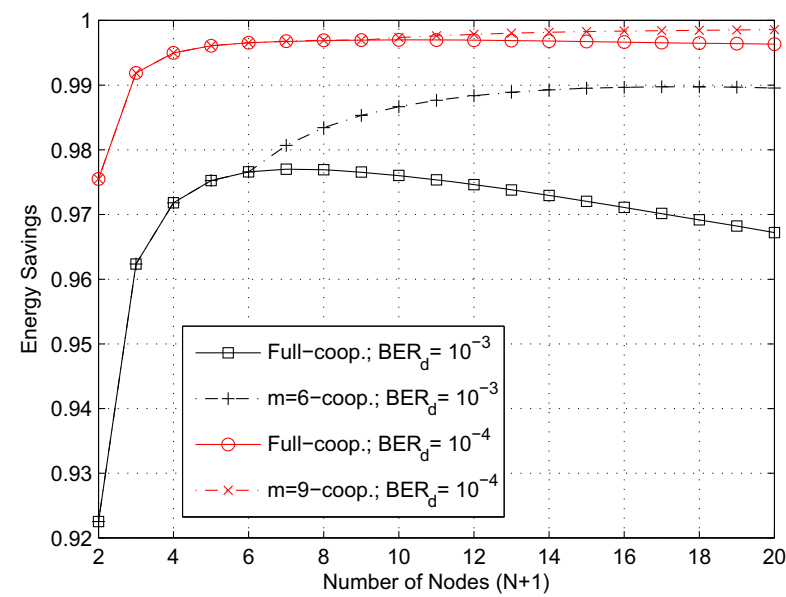

Fig. 3. The average energy savings curves versus the number of transmitting nodes $(N+1)$ relay networks employing full-cooperation and $m$-cooperation with two different $\mathrm{BER}_{d}$ constraints.

can be rewritten as

$$
P_{n}^{b} \leq 1-\left[\left(1-\mathrm{BER}_{\max }\right) \prod_{i=\max \{1, n-m\}}^{n-1}\left(1-P_{i}^{b}\right)\right] .
$$

To get an insight into the relationship between the endto-end BER $P_{N+1}^{b}$ and $\mathrm{BER}_{\max }$, the upper-bound on $P_{N+1}^{b}$ when full cooperation is used can be represented as $P_{N+1}^{b} \leq$ $1-\left(1-\mathrm{BER}_{\max }\right)^{2^{N}}$.

\section{NumERICAL ANALYSis}

\section{A. Performance Analysis: Regular Linear Networks}

In this section, we present some results to quantify the energy savings due to the proposed cooperative routing scheme. We consider a regular line topology where nodes are located at unit distance from each other on a straight line. The optimal non-cooperative routing in this network is to always send the information to the next nearest node in the direction of the destination. From (6), (28), and by assuming that $\sigma_{i, j}^{2}$ is proportional to the inverse of the distance squared, the total power required for non-cooperative transmission can be calculated as

$$
P_{T}(\text { noncoop })=(N+1) \frac{c \mathcal{N}_{0}}{2 g \mathrm{BER}_{\max }} .
$$

Since we restrict the cooperation to nodes along the optimal non-cooperative route, the total transmitted power for full- cooperation and $m$-cooperation in line networks can be obtained from (25) and (26), respectively. Thus, by replacing $\Psi(n)$ from (28) and $\sigma_{i, j}^{2}=1 /|i-j|^{2}$, we have

$$
P_{T}(\text { cooperative })=\sum_{n=1}^{N+1} n\left(\frac{c \prod_{i=1}^{n}(2 i-1)}{\mathrm{BER}_{\max }} \frac{\mathcal{N}_{0}^{n} n !}{2 g^{n}}\right)^{\frac{1}{n}},
$$




$$
\begin{aligned}
& P_{T}(m \text {-coop })=\sum_{n=1}^{m} n\left(\frac{c \prod_{i=1}^{n}(2 i-1)}{\mathrm{BER}_{\max }} \frac{\mathcal{N}_{0}^{n} n !}{2 g^{n}}\right)^{\frac{1}{n}} \\
&+(N-m+1) m\left(\frac{c \prod_{i=1}^{m}(2 i-1)}{\mathrm{BER}_{\max }} \frac{\mathcal{N}_{0}^{m} m !}{2 g^{m}}\right)^{\frac{1}{m}},
\end{aligned}
$$

In Fig. 2, we compare the achieved energy savings of the proposed cooperative routing with respect to the non-cooperative multihop scenario, in which satisfying the $\mathrm{BER}_{\max }$ at each step is used as a performance criteria. For the $\mathrm{BER}_{\max }=10^{-4}$, it can be observed that using the full cooperation scheme around $99 \%$ saving in energy is achieved when 4 relays are employed. Since the corresponding curve has an optimum performance when $N=4$, we consider the $m=5$ cooperation as an appropriate scheme. As it can be observed from Fig. 2, increasing the number of nodes in the network, $100 \%$ savings in energy is achievable. For the case of $\mathrm{BER}_{\max }=10^{-5}$, the same characteristics can be seen.

Fig. 3 demonstrates a lower-bound on the obtainable energy saving in line networks, when the required BER at the destination, i.e., $\mathrm{BER}_{d}$, should be satisfied. We use (31) to get a reliable power allocation at transmitting nodes to fulfil the required BER QoS at the destination node. For two cases of $\mathrm{BER}_{d}=10^{-3}$ and $\mathrm{BER}_{d}=10^{-4}$, vast amount of energy savings are obtainable. Since the maximum values of the curves corresponding to the full-cooperation routing occur when 5 and 8 relays are used, the 6-cooperation and 9-cooperation are used for $\mathrm{BER}_{d}=10^{-3}$ and $\mathrm{BER}_{d}=10^{-4}$ cases, respectively.

\section{B. Performance Analysis: Regular Grid Networks}

Fig. 4 shows a regular $4 \times 4$ grid topology with the source $s$ and destination $d$ located at the opposite corners. An $p \times p$ grid can be decomposed into many $2 \times 2$ grid. Without lose of generality, we assume that a transmission to a neighbor in vertical or horizontal direction has a cost of 1 unit. Under this assumption, in a $2 \times 2$ grid, a diagonal transmission has a cost of 2 units, equal to the cost of one horizontal and one vertical transmission. In an $p \times p$ grid, there are many noncooperative routes with an equal cost of $2(p-1)$ units. Fig. 4 shows two such routes for an $p \times p$ grid. We will base our analysis for the savings based on the stair-like noncooperative path shown in Fig. 4. Since nodes along the stair-like noncooperative path are closer together in comparison with other routes, it can be shown that this route has the highest energy saving for $(m>1)$-cooperation.

From (6), (28), and by assuming that $\sigma_{i, j}^{2}$ is proportional to the inverse of the distance squared, the total power required for non-cooperative transmission in a $p \times p$ grid can be calculated as

$$
P_{T}(\text { noncoop })=(2 p-2) \frac{c \mathcal{N}_{0}}{2 g \mathrm{BER}_{\max }} .
$$

Since we restrict the cooperation to nodes along the optimal non-cooperative route, the total transmitted power for full- cooperation and $m$-cooperation in grid networks can be obtained from (25). Thus, by replacing $\Psi(n)$ from (28) and $\sigma_{i, j}^{2}=1 /|i-j|^{2}$, we have

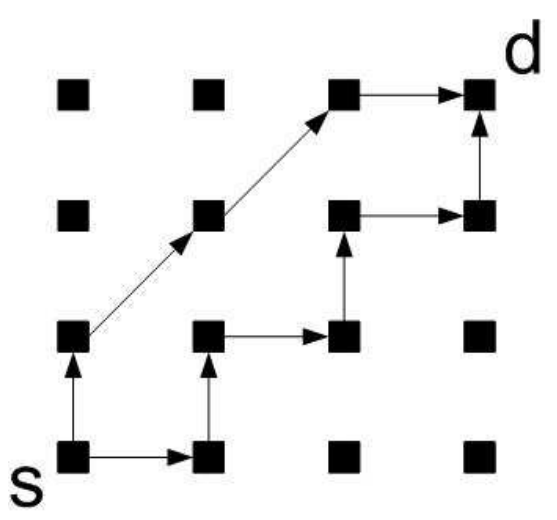

Fig. 4. A regular $4 \times 4$ grid topology with the source $s$ and destination $d$ where two possible routing paths are demonstrated.

$$
P_{T}(\text { cooperative })=\sum_{n=1}^{2 p-2} n\left(\frac{c \mathcal{N}_{0}^{n}}{2 g^{n} \mathrm{BER}_{\max }} \prod_{i=1}^{n}(2 i-1) D_{i}\right)^{\frac{1}{n}},
$$

where

$$
D_{i}=\left(\left\lceil\frac{i}{2}\right\rceil\right)^{2}+\left(\left\lfloor\frac{i}{2}\right\rfloor\right)^{2}
$$

where $\lfloor x\rfloor$ and $\lceil x\rceil$ denote the floor and ceil of $x$, respectively. Moreover, the total transmitted power for $m$-cooperation in grid networks can be obtained from (26) as

$$
\begin{aligned}
& P_{T}(m \text {-coop })=\sum_{n=1}^{m} n\left(\frac{c \mathcal{N}_{0}^{n}}{2 g^{n} \mathrm{BER}_{\max }} \prod_{i=1}^{n}(2 i-1) D_{i}\right)^{\frac{1}{n}} \\
& \quad+(2 p-m-2) m\left(\frac{c \mathcal{N}_{0}^{m}}{2 g^{m} \mathrm{BER}_{\max }} \prod_{i=1}^{m}(2 i-1) D_{i}\right)^{\frac{1}{m}},
\end{aligned}
$$

Now, we demonstrate the performance gain can be obtained using the proposed cooperation scheme. We consider a $p \times p$ grid network, with the stair-like noncooperative route.

In Fig. 5, we compare the achieved energy savings of the proposed cooperative routing with respect to the non-cooperative multihop scenario, in which satisfying the $\mathrm{BER}_{\max }$ at each step is used as a performance criteria. For the $\mathrm{BER}_{\max }=10^{-4}$, it can be observed that using the full cooperation scheme around $98 \%$ saving in energy is achieved when the network size of 9 is employed. Since the maximum amount of energy savings happens when network size is 9 , i.e., $3 \times 3$ grid network, $m=4$-cooperation is selected $(m=2 p-2=4)$. Thus, since the corresponding curve has an optimum performance for a network of size 9 , we consider the $m=4$ cooperation as an appropriate scheme. As it can be observed from Fig. 5, increasing the number of nodes in the network, $100 \%$ savings in energy is achievable. For the case of $\mathrm{BER}_{\max }=10^{-5}$, the same characteristics can be seen. Comparing the curves, it can be seen that by decreasing the 


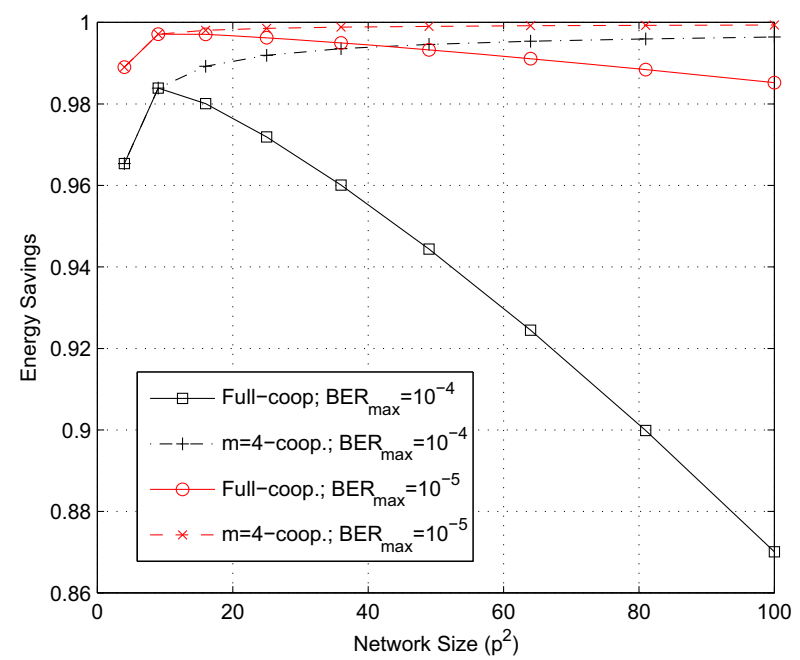

Fig. 5. The average energy savings curves versus the number nodes $p^{2}$ for $p \times p$ grid networks employing full-cooperation and $m$-cooperation with two different $\mathrm{BER}_{d}$ constraints.

value of BER QoS, more energy savings is achievable using the proposed cooperative routing.

Fig. 6 demonstrates a lower-bound on the obtainable energy saving in grid networks, when the required BER at the destination, i.e., $\mathrm{BER}_{d}$, should be satisfied. We use (31) to get a reliable power allocation at transmitting nodes to fulfil the required BER QoS at the destination node. For two cases of $\mathrm{BER}_{d}=10^{-3}$ and $\mathrm{BER}_{d}=10^{-4}$, enormous amount of energy savings are obtainable. Since the maximum amount of energy savings happens when network size is 9 , i.e., $3 \times 3$ grid network, $m=4$-cooperation is selected ( $m=2 p-2=$ $4)$. Thus, since the corresponding curve has an optimum performance for a network of size 9 , we consider the $m=4$ cooperation as an appropriate scheme for $\mathrm{BER}_{d}=10^{-3}$ and $\operatorname{BER}_{d}=10^{-4}$ cases.

\section{CONCLUSION}

In this paper, we formulated the problem of finding the minimum energy cooperative route for a wireless network under Rayleigh fading channel. We proposed a cooperative multihop routing for the purpose of power savings, constrained on a required BER at the destination. The calculated power coefficients are independent of instantaneous channel variation, and thus, can be used in practical wireless systems. We derive analytical results for line and grid network topologies. It is shown that energy savings of up to $100 \%$ are achievable in line and grid networks with a large number of nodes for $\mathrm{BER}=10^{-4}$ constraint at the destination.

\section{REFERENCES}

[1] J. H. Chang and L. Tassiulas, "Energy conserving routing in wireless adhoc networks," in Proc. IEEE Conference on Computer Communications (INFOCOM), (Monterey, CA), Mar. 2000.

[2] M. Younis, M. Youssef, and K. Arisha, "Energy-aware management for cluster-based sensor networks," IEEE Trans. Commun., vol. 43, no. 5, pp. 539-694, Dec. 2003.

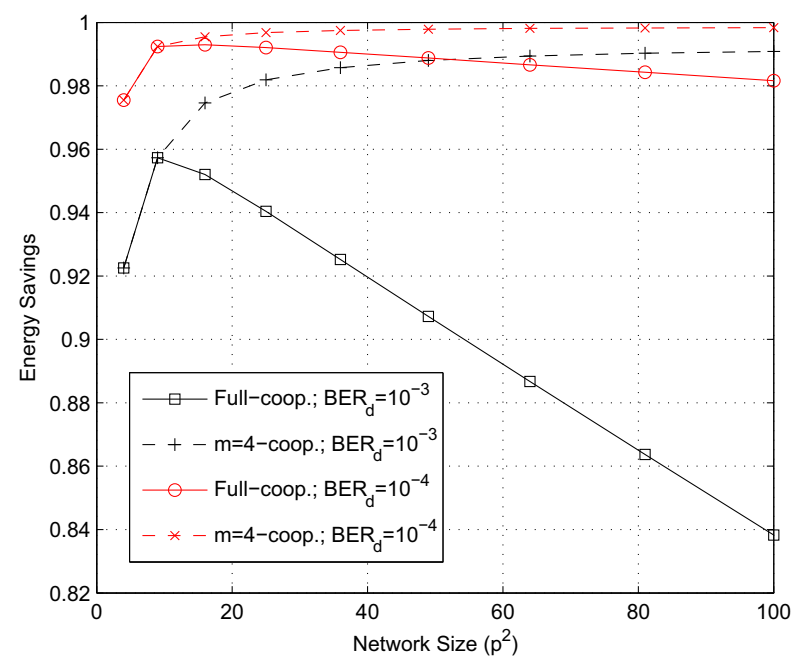

Fig. 6. The average energy savings curves versus the number nodes $p^{2}$ for $p \times p$ grid networks employing full-cooperation and $m$-cooperation with two different $\mathrm{BER}_{d}$ constraints.

[3] L. M. Feeney and M. Nilsson, "Investigating the energy consumption of a wireless network interface in an ad hoc networking environment," in Proc. IEEE Conference on Computer Communications (INFOCOM), vol. 3, pp. 1548-1557, Apr. 2001.

[4] B. Zhao and M. C. Valenti, "Practical relay networks: A generalization of hybrid-ARQ," IEEE J. Sel. Areas Commun., vol. 23, no. 1, pp. 7-18, Jan. 2005.

[5] S. Shakkottai, T. S. Rappaport, and P. C. Karlsson, "Cross-layer design for wireless networks," IEEE Commun. Mag., vol. vol. 41, no. 10, pp. 74-80, Oct. 2003.

[6] A. Sendonaris, E. Erkip, and B. Aazhang, "User cooperation diversity Part 1: System description; Part 2: Implementation aspects and performance analysis," IEEE Trans. Commun., vol. 51, pp. 1927-1938, Nov. 2003.

[7] J. N. Laneman and G. Wornell, "Distributed space-time coded protocols for exploiting cooperative diversity in wireless networks," in IEEE GLOBECOM 2002, vol. 1, (Taipei, Taiwan, R.O.C.), pp. 77-81, Nov. 2002.

[8] J. N. Laneman, D. Tse, and G. Wornell, "Cooperative diversity in wireless networks: Efficient protocols and outage behavior," IEEE Trans. Inform. Theory, vol. 50, no. 12, pp. 3062-3080, Dec. 2004.

[9] N. Vaidya, "Open problems in mobile ad-hoc networks," in Keynote talk at the Workshop on Local Area Networks, (Tampa, Florida), Nov. 2001.

[10] F. Li, K. Wu, and A. Lippman, "Energy-efficient cooperative routing in multi-hop wireless ad hoc networks," in Proc. IEEE Perform., Computing, and Commun. Conf. (IPCCC), pp. 215-222, 2006.

[11] J. Boyer, D. D. Falconer, and H. Yanikomeroglu, "Multihop diversity in wireless relaying channels," IEEE Trans. Commun., vol. 52, pp. 18201830, Oct. 2004.

[12] A. E. Khandani, J. Abounadi, E. Modiano, and L. Zheng, "Cooperative routing in static wireless networks," IEEE Trans. Commun., vol. 55, no. 11, pp. 2185-2192, Nov. 2007.

[13] A. S. Ibrahim, A. K. Sadek, W. Su, and K. R. Liu, "Cooperative communications with relay-selection: When to cooperate and whom to cooperate with?," IEEE Trans. Wireless Commun., vol. 7, no. 7, pp. 2814-2827, Jul. 2008.

[14] M. K. Simon and M.-S. Alouini, Digital Communication over Fading Channels: A Unified Approach to Performance Analysis. New York, NY: Wiley, 2005.

[15] A. Ribeiro, A. Cai, and G. B. Giannakis, "Symbol error probablity for general cooperative links," IEEE Trans. Wireless Commun., vol. 4, pp. 1264-1273, May 2005.

[16] S. Boyd and L. Vandenberghe, Convex Optimization. Cambridge: Cambridge Univ. Press, 2004.

[17] T. Wang, A. Cano, G. B. Giannakis, and J. N. Laneman, "Highperformance cooperative demodulation with decode-and-forward relays," IEEE Trans. Commun., vol. 55, no. 6, pp. 830-841, Apr. 2007. 